8

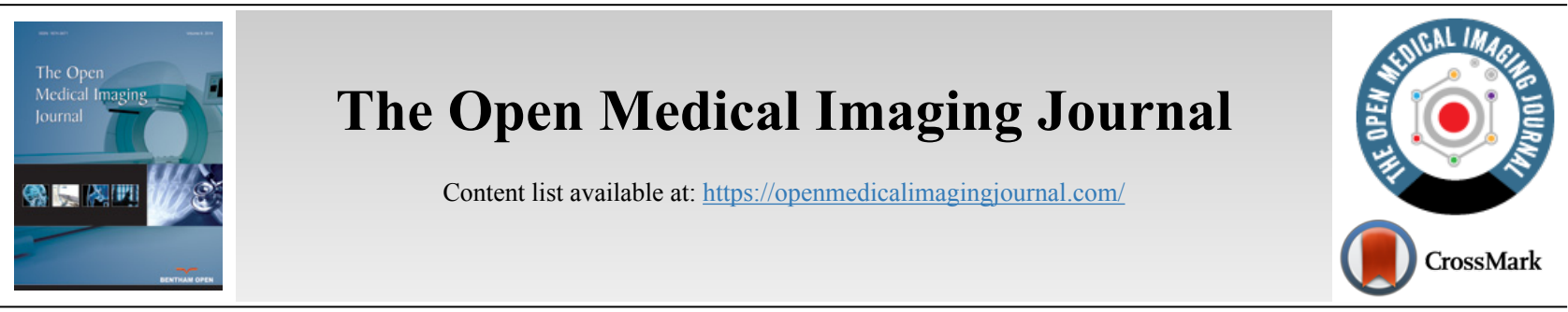

RESEARCH ARTICLE

\title{
Ripplet-Transform-based Cycle Spinning Denoising and Fuzzy-CLA Segmen- tation of Retinal Images for Accurate Hard Exudates and Lesion Detection
}

\author{
Hadi Chahkandi Nejad ${ }^{1, *}$, Mohsen Farshad ${ }^{2}$, Tahereh Farhadian ${ }^{3}$ and Roghayeh Hosseini ${ }^{4}$ \\ ${ }^{I}$ Department of Electrical Engineering, Birjand Branch, Islamic Azad University, Birjand, Iran \\ ${ }^{2}$ Department of Electrical and Computer Engineering, University of Birjand, Birjand, Iran \\ ${ }^{3}$ Faculty of Medical Sciences, Birjand Branch, Islamic Azad University, Birjand, Iran \\ ${ }^{4}$ Department of Electrical Engineering, Mashhad Branch, Islamic Azad University, Mashhad, Iran
}

\begin{abstract}
:
Aims:

Digital retinal images are commonly used for hard exudates and lesion detection. These images are rarely noiseless and therefore before any further processing they should be underwent noise removal.

Background:

An efficient segmentation method is then needed to detect and discern the lesions from the retinal area.

Objective:

In this paper, a hybrid method is presented for digital retinal image processing for diagnosis and screening purposes. The aim of this study is to present a supervised/semi-supervised approach for exudate detection in fundus images and also to analyze the method to find the optimum structure.

Methods:

Ripplet transform and cycle spinning method is first used to remove the noises and artifacts.

Results:

The noises may be normal or any other commonly occurring forms such as salt and pepper. The image is transformed into fuzzy domain after it is denoised.

Conclusion:

A cellular learning automata model is used to detect any abnormality on the image which is related to a lesion. The automaton is created with an extra term as the rule updating term to improve the adaptability and efficiency of the cellular automata.Three main statistical criteria are introduced as the sensitivity, specificity and accuracy. A number of 50 retinal images with visually detection hard exudates and lesions are the experimental dataset for evaluation and validation of the method.
\end{abstract}

Keywords: Digital retinal images, Hard exudates and lesions detection, Denoising, Fuzzification concept, Cellular Learning Automata, Statistical evaluation parameters, Ripplet transform.

\begin{tabular}{l|l|l|r} 
Article History & Received: June 17, 2019 & Revised: November 07, 2019 & Accepted: November 11, 2019
\end{tabular}

\section{INTRODUCTION}

In ophthalmology, the automatic detection of blood vessels, as well as the detection of the optic disc, may be of consi* Address correspondence to this author at the Department of Electrical
Engineering, Islamic Azad University Birjand, Birjand, Iran;

Tel: +989155623083; E-mail: Hchahkandin@iaubir.ac.ir derable interest in computer assisted diagnosis. Detecting and counting lesions, in the human retina such as micro-aneurysms and exudates, is a time-consuming task for ophthalmologists and open to human error. That is why much effort has been made to detect lesions in the human retina automatically [1]. Diabetic Retinopathy (DR) is a visual complication of diabetes, 
which has become the most common cause of visual impairment and new-onset blindness among people of working age in industrialized countries [2,3]. Due to its prevalence and clinical significance, the research community has attempted to improve its diagnosis and treatment by developing algorithms to perform retinal image analysis [4], fundus image enhancement [5], and monitoring [6]. Of special significance are automatic image analysis algorithms designed to detect Hard Exudates (HEs) [7]. HEs have been found to be the most specific markers for the presence of retinal oedema, the major cause of visual loss in non-proliferative forms of DR [2]. Additionally, HEs are one of the most prevalent lesions during the early stages of DR [2].

In many applications, image denoising is a tool by which a good estimate of the original image from noisy states is provided. An ideal image denoising technique makes it possible to eliminate as much random additive noise as possible while maintaining key image characteristics, such as edges and texture. Among the many methods proposed for this purpose, i.e. image denoising, due to their high ability in the sparse representation of signals, transform based methods (e.g., wavelets) [8] are dominant in this application.

Automatic algorithms for HE detection are required in a variety of applications, including the design of complete systems for automatic processing of retinal images. Several techniques have been developed for HE detection in fundus images based on a variety of techniques [7]. These techniques include the usage of image contrast and brightness analysis [9 - 15], Bayesian classifiers [16, 17], and neural networks [18, 19].

Zhang et al. [20] used local contrast enhancement and Fuzzy C-means Clustering (FCMC) in Luv color space to segment candidate bright exudates areas. However, the main difficulty with FCMC is determining the number of clusters to use. Walter et al. [21] applied mathematical reconstruction to detect contours typical of exudates. This technique achieved predictive and sensitivity values of $92.4 \%$ and $92.8 \%$ with a set of 15 abnormal retinal images. However, this technique did not discriminate exudates from cotton wool spots. Gardner et al. [22] used the backpropagation neural network for the segmentation of exudates. Comparing the results of this method with those of an expert ophthalmologist, the method achieved a sensitivity and specificity of $88.4 \%$ and $83.5 \%$, respectively, for the detection of exudates. A drawback of this method was that it did not work well on poor quality images.

Most of the previous techniques have based on retinal features being clearly visible on acquired imagery. Acquiring clearly visible retinal features takes time and it is uncomfortable for patients. Moreover, sometimes, there are a number of exudates with different sizes apart or stuck, which require a model-based method for detection. Therefore, in this paper, the cellular learning automata in the fuzzy domain is used for automated and accurate detection of exudates on retinal images. The ultimate aim was to develop an application for automatic detection of exudates, to provide decision support and to reduce workloads for expert ophthalmologists by a supervised method, which can also act as an automation system.
Cellular Automata (CA) consist of a regular grid of cells, each of which can be in only one of a finite number of possible states. The state of a cell is determined by the previous states of a surrounding neighborhood of the cells and is updated synchronously in discrete time steps. The identical rule contained in each cell is essentially a finite state machine, usually specified in the form of a rule table with an entry for every possible neighborhood configuration of states. Cellular automata are discrete dynamical systems, and they have been found useful for simulating and studying phenomena such as ordering, turbulence, chaos, symmetry-breaking, etc., and have had wide application in modeling systems in areas such as physics, biology and sociology. One of the advantages of CAs is that, although each cell generally only contains a few simple rules, the combination of a matrix of cells with their local interaction leads to more sophisticated emergent global behavior. That is, although each cell has an extremely limited view of the system (just its immediate neighbors), localized information is propagated at each time step, enabling more global characteristics of the overall CA system [23].

In this paper, a denoising method based on ripplet transform is first presented. Fuzzified cellular learning automata are then introduced for the segmentation of denoised retinal images. A retinal image dataset consisting of 50 retinal images with hard exudates is used to assess the proposed method. The paper is organized as follows: Section 2 presents the dataset and the methodology results are given in Section 3 and in Section 4, the results are discussed. Finally, some conclusions are drawn in Section 5.

\section{MATERIALS AND METHODS}

\subsection{Image Database}

The main dataset was a subset of the STARE Project's dataset [27]. The subset contained 81 retinal images for evaluating the proposed exudates detection method. The images were captured using a TopCon TRV-50 fundus camera at $35^{\circ}$ field-of-view (FOV), and subsequently digitized at $605 \times$ 700, 24-bits pixel. The dataset was used for both the evaluation of the proposed method and also comparison to some other methods. The dataset consisted of a total of 50 color retinal images which were taken without pupil dilation with a KOWA-7 non-mydriatic retinal camera with a $45^{\circ} \mathrm{FOV}$. The image size was $768 \times 576$ pixels at 24 bits per pixel in RGB format. All retinal images analyses were performed on a Core2 Duo 2.2 GHz Laptop using MATLAB 7.6.0 for all implementations.

\subsection{Denoising Digital Retinal Images}

The processing of digital retinal images is only possible by the discrete form of the transform methods. Hence, the discrete form of ripplet transform is applied to digital retinal images for denoising purposes. Analogously to the discrete curvelet transform, the discretization of continuous ripplet transform is based on the discretization of the parameters of ripplets, i.e. $a, \vec{b}$ and $\theta$. To this end, $a$ is sampled at the dyadic interval while the position parameter $\vec{b}$ and the rotation parameter $\theta$ are sampled at the equal-spaced intervals. The discrete versions 
of $a, b_{k}$ and $\theta$ are denoted by $a_{j}, \overrightarrow{b_{k}}, \theta_{i}$ which satisfy that, (Eq.1)

$$
\begin{aligned}
& a_{j}=2^{-j} \\
& \vec{b}_{k}=\left[c \cdot 2^{-j} \cdot k_{1}, 2^{-j / d} \cdot k_{2}\right]^{\mathrm{T}} \\
& \theta_{l}=\frac{2 \pi}{c} \cdot 2^{-[j(l-1) / d]} \cdot l
\end{aligned}
$$

in which (Eq.2)

$$
\vec{k}=\left[k_{1}, k_{2}\right]^{\mathrm{T}}
$$

and operator $T$ denotes the transpose of a vector, $j, \mathrm{k} 1, \mathrm{k} 2$, $l \in \mathbb{Z}, m, n \in N$ (usually $\mathrm{m}$ and $\mathrm{n}$ are both primes), $d \in \mathbb{R}$ and $d=m / n \quad$ [8]. Therefore, the frequency response of discrete ripplet function can be represented as, (Eq.3)

$$
\hat{\rho}_{j}(r, \omega)=\frac{1}{\sqrt{c}} a^{\frac{m+n}{2 n}} W\left(2^{-j} \cdot r\right) V\left(\frac{1}{c} \cdot 2^{-\left[j \frac{m-n}{n}\right]} \cdot \omega-l\right)
$$

In which $\mathrm{W}$ and $V$ satisfy the following admissibility condition (Eqs.4 and 5):

$$
\begin{gathered}
\sum_{j=0}^{+\infty}\left|W\left(2^{-j} \cdot r\right)\right|^{2}=1 \\
\sum_{j=0}^{+\infty}\left|V\left(\frac{1}{c} \cdot 2^{-[j(j-1) / d]} \cdot \omega-l\right)\right|^{2}=1
\end{gathered}
$$

In the frequency domain, the 'wedge' of the ripplet function can be represented as (Eq.6):

$$
H_{j, l}(r, \theta)=\left\{2^{j} \leq|\mathrm{r}| \leq 2^{2 j},\left|\theta-\frac{\pi}{c} \cdot 2^{-[j(l-1) \mathrm{d}]} l\right| \leq \frac{\pi}{2} 2^{-j}\right\}(\mathbf{6})
$$

Finally, for an arbitrary image $f\left(\mathrm{n}_{1}, \mathrm{n}_{2}\right)$ with size $L \times M$ the discrete ripplet coefficients will be in the form of (Eq.7):

$$
R_{j, \vec{k}, l}=\sum_{n_{1}=0}^{L-1} \sum_{n_{2}=0}^{M-1} f\left(n_{1}, n_{2}\right) \overline{\rho_{j, \vec{k}, l}\left(n_{1}, n_{2}\right)}
$$

The image can be reconstructed from the coefficients $R_{j, \vec{k}, l}$ through inverse discrete ripplet transform (Eq.8):

$$
\tilde{f}\left(\mathrm{n}_{1}, \mathrm{n}_{2}\right)=\sum_{j} \sum_{\vec{k}} \sum_{l} R_{j, \vec{k}, l} \rho_{j, \vec{k}, l}\left(n_{1}, n_{2}\right)
$$

In the shift variant transforms such as ripplet transform, the pseudo-Gibbs phenomenon results in appearing artifacts in the singularities around the edges of the reconstructed image. To alleviate these artificial edges, traditional methods use a strategy in which the image is shifted to change the position of the discontinuity point [8]. After processing (denoising in our case) shifted image, the inverse shifting was applied to the shifted image.

Assume $f\left(\mathrm{n}_{1}, \mathrm{n}_{2}\right)$ be the noisy image, $f\left(\mathrm{n}_{1}, \mathrm{n}_{2}\right)$ be the estimation of noise-free image, $\mathrm{R}$ and $\mathrm{R}-1$ denote the ripplet transform and the inverse ripplet transform, respectively, $d_{x}$, and is the shift distance along the horizontal direction $\mathrm{x}$ and vertical direction $y$, respectively [13]. Thus, if the image is shifted with a certain distance, $\mathrm{d}_{\mathrm{x}, \mathrm{y}}$ the denoising strategy using the cycle spinning method can be expressed as follows (Eq.9).

$$
\hat{f}\left(\mathrm{n}_{1}, \mathrm{n}_{2}\right)=d_{-x,-y}\left(R^{-1}\left(\operatorname{Denosing}\left(R\left(d_{x, y}\left(f\left(\mathrm{n}_{1}, \mathrm{n}_{2}\right)\right)\right)\right)\right)\right)
$$

where denoising is the process described in the previous section. However, this strategy works, when the number of discontinuous points in an image is small, whereas the shift may cause serious pseudo-Gibbs phenomenon in the area of other discontinuous points. Therefore, it is hard to find a proper shift distance which can cope with all of discontinuous points in the image. Thus, the cycle spinning method is proposed to tackle this problem in denoising of images.

Given $x \in X$ and $y \in Y$ which indicate a set of shift arranges $X=\{x 1, x 2, \ldots, x e 1\}$ in vertical direction and $Y=\{y 1, y 2, \ldots, y e 2\} \quad$ in horizontal direction, and considering that all the $\mathrm{x}$ and $\mathrm{y}$ should be less than the columns and the rows of the image, respectively, averaging the denoised image after a series of the cycle spinning, gives the denoised image in which the pseudo-Gibbs phenomenon is alleviated. The denoising method utilizing the cycle spinning can be described by the following formula (Eq.10).

$\hat{f}\left(\mathrm{n}_{1}, \mathrm{n}_{2}\right)=$ Mean $_{x \in X, y \in Y} d_{-x,-y}\left(R^{-1}\left(\operatorname{Denosing}\left(R\left(d_{x, y}\left(f\left(\mathrm{n}_{1}, \mathrm{n}_{2}\right)\right)\right)\right)\right)\right)$

\subsection{Transformation of Retinal Images into Fuzzy Domain}

Let us consider an image of size $M \times N$ pixels, having $L$ gray levels $g$ ranging from 0 to $L-1$. The image $\gamma$ can be viewed as an array of fuzzy singletons [19]. Each element of the array is the membership value $\mu_{\gamma i}\left(g_{x y}\right)$ of the gray level $g_{x y}$, corresponding to the $(x, y)$ pixel, regarding an image property such as brightness, edginess, homogeneity, etc. Using the fuzzy sets notation image $\gamma$ can be represented as (Eq.11):

$\gamma^{i}=\left\{\frac{\mu_{\gamma^{i}}\left(g_{x y}\right)}{g_{x y}} \mid i=0,1, \ldots, M-1, j=0,1, \ldots, N-1\right\}$

To enhance the gray levels across the edges and to improve the contrast of the image, gray levels of the pixels are normalized to unit interval $[0,1]$ corresponding to the whole gray level range [0,255]. The primary motion matrix is obtained by the calculation of $\Omega$ function over $\check{\delta} \times \check{\delta}$ neighborhood windows around all pixels as (Eqs.12-14), 


$$
\begin{array}{r}
\Omega^{\mathrm{i}}(\mathrm{x}, \mathrm{y})=\left\{\begin{array}{cc}
\nabla^{\mathrm{i}, \mathrm{i}-1} \times \nabla^{\mathrm{i}, 9^{\mathrm{i}}} & \text { if } \frac{1}{\check{\delta}^{2}} \sum \mid \gamma^{i}(x-1: x+1, y-1: y+1) \\
0 & -\gamma^{i-1}(x-1: x+1, y-1: y+1) \mid>T_{1}
\end{array}\right. \\
\text { otherwise, }
\end{array}
$$

where

$$
\begin{array}{r}
\nabla^{\mathrm{i}, \mathrm{i}-1}=\frac{1}{\check{\delta}^{2}} \sum \mid \gamma^{i}(x-1: x+1, y-1: y+1) \\
-\gamma^{i-1}(x-1: x+1, y-1: y+1) \mid
\end{array}
$$

and

$$
\begin{array}{r}
\nabla^{\mathrm{i}, \vartheta^{\mathrm{i}}}=\frac{1}{\check{\delta}^{2}} \sum \mid \gamma^{i}(x-1: x+1, y-1: y+1)-\frac{1}{i-1} \\
\sum_{j=1: i-1} \gamma^{j}(x-1: x+1, y-1: y+1) \mid
\end{array}
$$

Matrix $\Omega^{i}$ is obtained as the primary motion detected in $i^{t h}$ image of the image set $\Gamma$. Parameter $T_{1}$ is the threshold value for motion detection in corresponding $\breve{\delta} \times \check{\delta}$ neighborhood windows in image sequence $i^{t h}$ and $(i+1)^{\text {th }}$. Coefficient $\nabla^{\mathrm{i}, \mathrm{i}-1}$ means the first order absolute gradient of the sequent images $\gamma^{i}$ and $\gamma^{i-1}$ and $\nabla^{\mathrm{i}, 9^{\mathrm{i}}}$ depicts the first order gradient of the image relative to the average of the previous images $\gamma^{1}: \gamma^{i-1}$ Multiplication of the two terms in Eq. (2) guarantees both of these statements: (a) difference with the previous image implies motion and (b) slight differences with the average of images represent vibration and undesired motions (e.g., shadows, camera shaking or local illumination variations).

The matrix $\Omega^{i}$ undergoes normalization in the [minimum,maximum] range as, (Eq.15),

$\Omega^{i} \rightarrow \underline{\Omega}^{i}=\left(\Omega^{i}-\min \left(\Omega^{i}\right)\right) \times \frac{L-1}{\max \left(\Omega^{i}\right)-\min \left(\Omega^{i}\right)}$

Therefore matrix $\Omega^{i}$ is normalized to the range $[0,255]$. Eq. (5) also represents a defuzzification formula though some elements of the matrix are out of the unit interval. Now, Cellular Learning Automata is used to detect the object of interest in the primary motion matrix $\Omega^{i}$ which has been converted to grayscale image format.

\subsection{Retinal Image Fuzzy Automaton}

A retinal image fuzzy automaton in the Fuzzy Cellular Learning Automata is created as, (Eq.16)

$\mathrm{A}=($ States; Neighborhood; Rules; Parameters; Update $)$

$S:$ States as $\left\{S_{i}^{t}\right\}$ in the range $\mathrm{S}_{\mathrm{i}}^{\mathrm{t}} \in[\mathrm{min}, \max ], S_{i}^{t}: i^{\text {th }}$

State at time $t$ where:

$$
\begin{aligned}
& S_{i}^{t}:\left\{\mu_{i}^{t} \in[0,1] \mid \exists \text { a one }- \text { to }- \text { one function } F(.)\right. \\
& \left.\therefore \forall \mu_{i}^{t} \rightarrow \exists F\left(\mu_{i}^{t}\right) \in[\min (S), \max (S)]\right\} \\
& \text { and }
\end{aligned}
$$

$$
S=\left\{S_{:}^{t} \mid t=1: \tau\right\}
$$

N: Neighborhood as $\delta \times \delta \quad$ Extended Moore Neighborhoods:

$$
\begin{gathered}
N^{\delta}(x, y):\left\{\left(x-\left[\frac{\delta}{2}\right]: x+\left[\frac{\delta}{2}\right], y-\left[\frac{\delta}{2}\right]: y+\left[\frac{\delta}{2}\right]\right)\right\} \\
\text { where } \delta \in\{2 \mathrm{k}+1 \mid \mathrm{k} \in \mathbb{N}\},
\end{gathered}
$$

\section{R: Rules as}

$R:\left\{p_{i} \rightarrow q_{i} \mid p_{i} \& q_{i}\right.$ are logical terms and $\left.i=1: N_{R}\right\}$

which are once defined by the user according to a set of training samples.

$P=(\vartheta, \xi, T):$ Parameters as $\vartheta:$ score, $\xi:$ penalty and $T:$ similarity threshold where $T=\left\{\rho^{i}\right\}_{\mathrm{i}=1: \mathrm{N}_{\mathrm{R}}}$. U: Updating functions for learning rules

$$
\mathrm{U}: \Xi(\mathrm{R} \rightarrow \widehat{\mathrm{R}})
$$

States in the automaton correspond to decision variables of the problem. The rules are set as transition functions (Eq.17):

$$
\mathrm{R}: \Psi\left(S^{t}(x, y) \rightarrow S^{t+1}(\hat{x}, \hat{y})\right)
$$

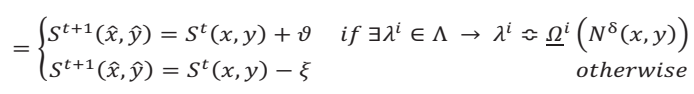

where $\Psi($.$) is the transition function,$ $S^{t}(\mathrm{x}, \mathrm{y})$ and $S^{t+1}(\hat{x}, \hat{y}) \quad$ correspond to the current state and the next state, $\vartheta$ and $\xi$ are the score and penalty values, respectively, $\Lambda=\left\{\lambda^{i}\right\}_{i=1: N_{\Lambda}}$ is the set of rules and the term $\lambda^{i} \approx \Omega^{i}($.$) implies the conditional rule applying. The$ condition under which the appropriate rule is selected and then applied can be logically expressed as (Eq.18):

$$
\lambda^{i} \approx \underline{\Omega}^{i}=\left\{\begin{array}{l}
1 \quad \text { if } \frac{1}{\delta^{2}} \times \sum_{\text {otherwise }} \frac{\left|\lambda^{i}-\underline{\Omega}^{i}\right|}{L-1}>\rho^{i} \\
0
\end{array}\right.
$$

where $\rho^{i}$ is the accordance factor for the $i^{\text {th }}$ rule. The conditional term of the above equation is similar to searching within a look-up table, which comprises a set of predefined state rules. Threshold $\rho^{i}$ selection directly specifies the sensitivity to the rules selection and to the state changes. Very low $\rho^{i}$ values lead to significant score assignments and it may result in enlarging the state values and also vice versa. Therefore, this parameter can be justified in each time interval 
of the cellular automata and this updating process leads to a learning scheme. Threshold value $\rho^{i}$ is updated in each cycle according to this formula (Eq.19):

$$
U: \rho^{i}=\left\{\begin{array}{c}
\rho^{i}+\sigma \quad \text { if } \eta>\bar{\eta} \\
\rho^{i}-\sigma \quad \text { otherwise }
\end{array}\right.
$$

where $\sigma$ is the updating additive value ranged between 0.001 and 0.1 . Parameter $\sigma$ is the ratio of state overall variation in three sequent cycles in cellular automata and $\eta$ is a threshold value for $\bar{\eta}$. For simplicity $\eta$. can be initially set as 0.6 and $\bar{\eta}$ is randomly selected in the unit interval $[0,1]$. Eq. (3) makes the threshold value $\eta$ larger if the rule selection sensitivity and the score assignment raise significantly since larger $\rho^{i}$ values limit the score assignments (and YIFHYHUD). The evolution cycle of the Cellular Learning Automata goes forward according to the learning rule. Score and penalty parameters can also be updated in each cycle but they are preferred to be set as low as possible initially and the convergence to be reached in a long time. Applying appropriate learning functions to the rules will guarantee the convergence and goal tracing capability of the model.

\subsection{Segmentation of Retinal Images}

For lesion detection in retinal images, a binary image comprising two segments of the healthy area of the retina as background and the detected lesion(s) as the foreground is desired. Therefore, since after the cycle $t=\tau$, the resulting image is in the fuzzy domain and its pixels are in the unit interval, a non-sensitive threshold $\varsigma$ ranged between 0.1 and 0.9 is selected to produce a binary image $I_{b}$ as:

$$
\begin{aligned}
& \text { for all }(\hat{x}, \hat{y}) \in S^{t}(.) \\
& \qquad \begin{array}{l}
\text { if } S^{t}(\hat{x}, \hat{y}) \geq \varsigma \rightarrow I_{b}(\hat{x}, \hat{y})=1 \\
\quad \text { esle } I_{b}(\hat{x}, \hat{y})=0 \\
\quad \text { endif }
\end{array} \\
& \text { endfor }
\end{aligned}
$$

\subsection{Segmentation Quantitative Criteria}

To report the performance of the classifier, the three common criteria sensitivity, specificity and accuracy are reported and calculated as follows (Eqs.20-22):

$$
\begin{aligned}
& \text { Sensitivity }(\%)=\frac{T P}{T P+F N} \times 100 \\
& \text { Specificity }(\%)=\frac{T N}{T N+F P} \times 100 \\
& \text { Accuracy }(\%)=\frac{T P+T N}{T P+F P+F N+T P} \times 100
\end{aligned}
$$

where the abbreviations and their meanings are summarized in Table $\mathbf{1}$. The sensitivity results describe the percentage of correctly detected object's pixels. The specificity result demonstrates the percentage of incorrect assignments of the pixels and the accuracy shows the validity of the assignments. The higher accuracy percentage reveals the higher veracity of results.

\section{SIMULATION RESULTS}

A number of 50 retinal images with hard exudates and lesion detected visually are considered as the dataset to evaluate the proposed segmentation method. Implementations of the proposed method were done in MATLAB software using an image processing toolbox. All codes were developed in MATLAB M-files, while a set of the images is copied in the same directory as the M-file. The program consists of four main parts: denoising step, initialization part, segmentation method and image binarization and assessment part.

In our experiments, we first evaluated the methods to find the optimum parameter values and the most suitable structure. Then, the results of the retina exudates detection for the proposed method are compared to the results of some other methods in recent literature. All detections were executed for lesion based images with no limitations in the number and regions of the lesions. A lesion is considered a true positive (TP) if it overlaps at least in part with the ground-truth; a false negative (FN) if no corresponding lesion is found in the automatic segmentation; a false positive (FP) if an exudate is found in the automatic segmentation, but no corresponding lesion has been manually segmented. In the evaluation of the segmentation, true negatives (TN) are the true assignments to the non-lesion regions.

Table 1. The abbreviations used in formula (20), (21) and (22).

\begin{tabular}{|c|c|c|}
\hline Abbreviation & Stands for & Description \\
\hline TP & True Positive & Pixels correctly detected as an object \\
\hline FP & False Positive & Pixels incorrectly marked as an object \\
\hline TN & True Negative & Pixels correctly marked as background \\
\hline FN & False Negative & $\begin{array}{c}\text { Pixels incorrectly labeled as backgrou- } \\
\text { nd }\end{array}$ \\
\hline
\end{tabular}

In the first part of the experiments, the effects of the score and penalty values for two iteration values were studied. All runs have been done for two neighborhood sizes of $3 \times 3$ and $5 \times 5$. Samples of the original retinal images with hard exudates are shown in (Figs. 1-3) show noisy images and the images denoised by the ripplet transform-based denoising method presented in this paper. Segmented images of retinal images are shown in Fig. (4). The images in Fig. (4) correspond to the implementation of the proposed method with a marginally optimum set of parameters, while their numerical results are given in Table 2 . The results of statistical analysis on the score and penalty assignments are given in Table $\mathbf{2}$ while the proposed method is used with and without the denoising process. It is shown that the optimum score and penalty values stand about 0.01 and 0.02 while the product of score/penalty values and the number of iterations is important. The denoising process improves the statistical parameters of about $4-6 \%$. It is also shown that for our dataset images, the neighborhood size of $5 \times 5$ which is an extended Moore neighborhood, leads to better results as compared to the simple Moore neighborhood.

Table 3 shows the results of a brief comparison between the Fuzzy cellular automata and fuzzy cellular learning automata. For this part of the experiment, the rules are updated for two values of $\bar{\eta} \& \sigma$. Parameter $\bar{\eta}$ affects the rate of score 
assignment and $\sigma$ determines the threshold for rule selection. Optimum values for $\bar{\eta} \& \sigma$ are found as $\bar{\eta}=0.5 \& \sigma=0.02$. Different similarity thresholds for rule selection from 0.5 to 0.95 are used to figure out the detected exudates and it has been shown that low threshold values result in enlarged detected regions and YFHYHWD It happens because low threshold values cause higher rates of rule selection and applying rules relates to the score assignment and exudates configuration. The similarity threshold value $\rho \sim 0.8-0.85$ equally for all rules has been found optimum.

The final state of the FCLA iterative method gives out a retina image with segmented exudates. Based on the number of iterations, defined rules and the values of score and penalty, the final state may be an image in the fuzzy domain and its pixels ranged in the unit interval. Hence, a threshold parameter for image binarization $s$ is proposed to assign the pixels of the images either 0 or 1 according to their fuzzy value. If appropriate rules are defined and suitable values for $\vartheta, \xi \& \tau$ are set, the sensitivity to parameter $\varsigma$ is too low. Then the values in the range $[0.3,0.9]$ will lead to slightly different binary images. The differences between the statistical parameters for three different values of $\varsigma$ are demonstrated in Table 4.

Comparison to some other methods is performed in terms of the statistical parameters. To evaluate the efficiency of the proposed FCLA method, the results of the six methods of references [15, 19 - 22], K-Nearest Neighbor method are listed in Table 5. The results for the proposed method are attained with the optimum parameters found in the previous parts of the experiment. These parameters are $N^{\delta}=5 \times 5, \vartheta=0.01, \xi=0.01, \tau=50, \bar{\eta}=0.5, \sigma=0.02, \rho=$ $0.8 \& \varsigma=0.5$. It is seen that the proposed method with its optimum parameters can detect the exudates in retina images accurately with high sensitivity and specificity.

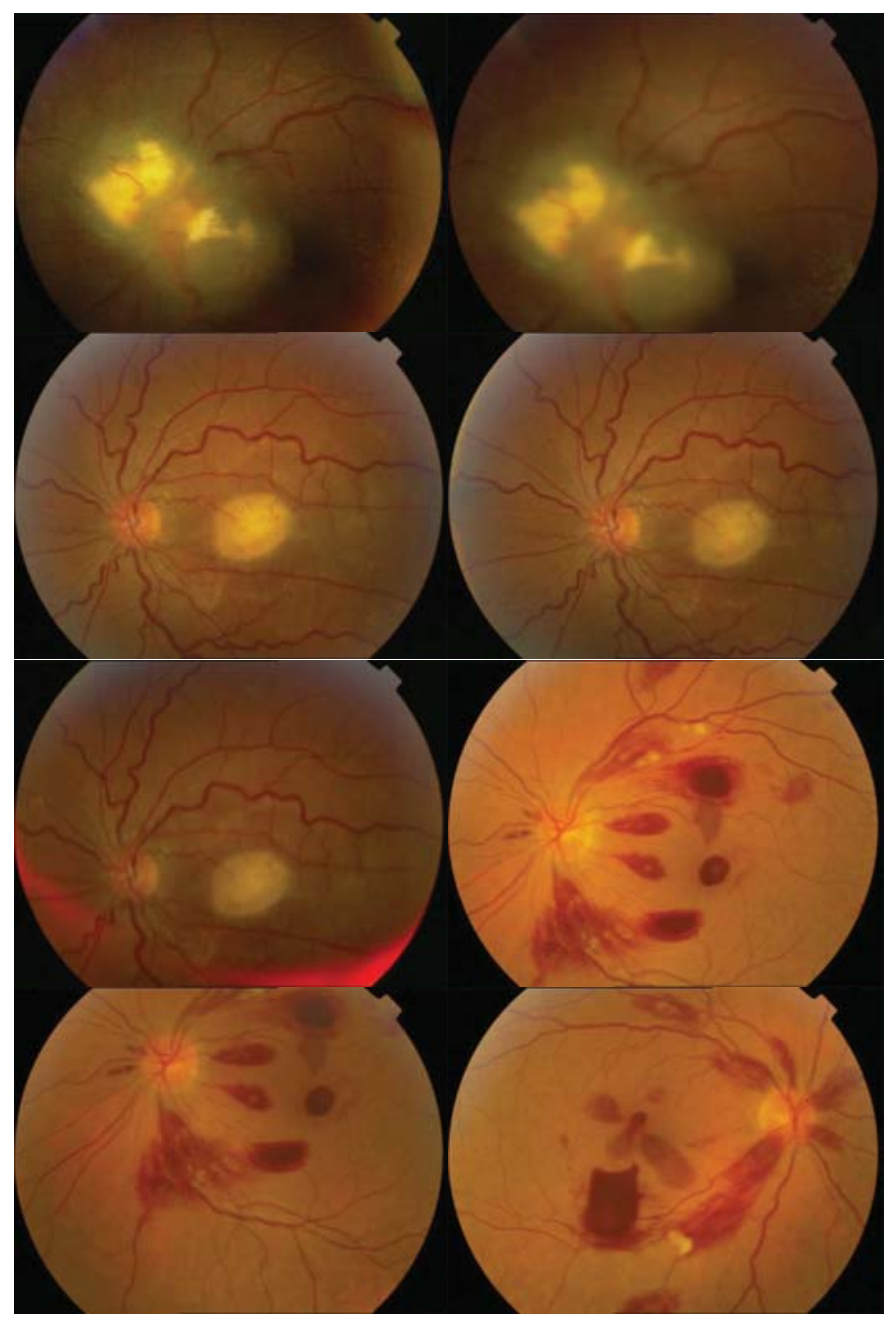

Fig. (1). Original Retinal Images with hard exudates. 

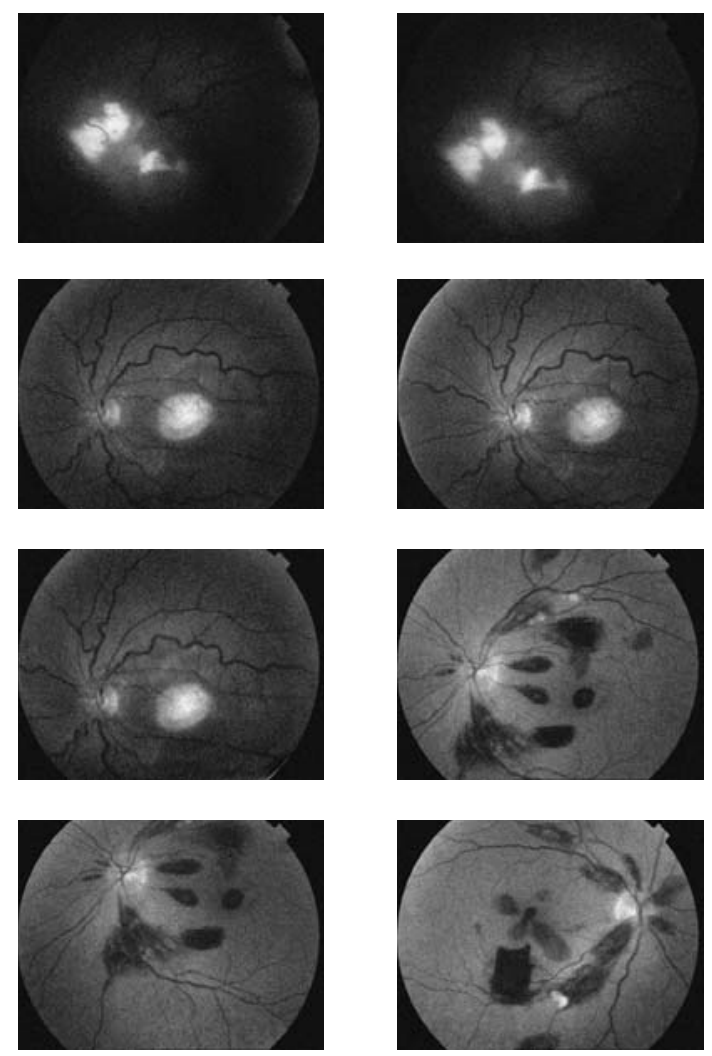

Fig.(2). Noisy Retinal Images with hard exudates (salt and pepper noise).
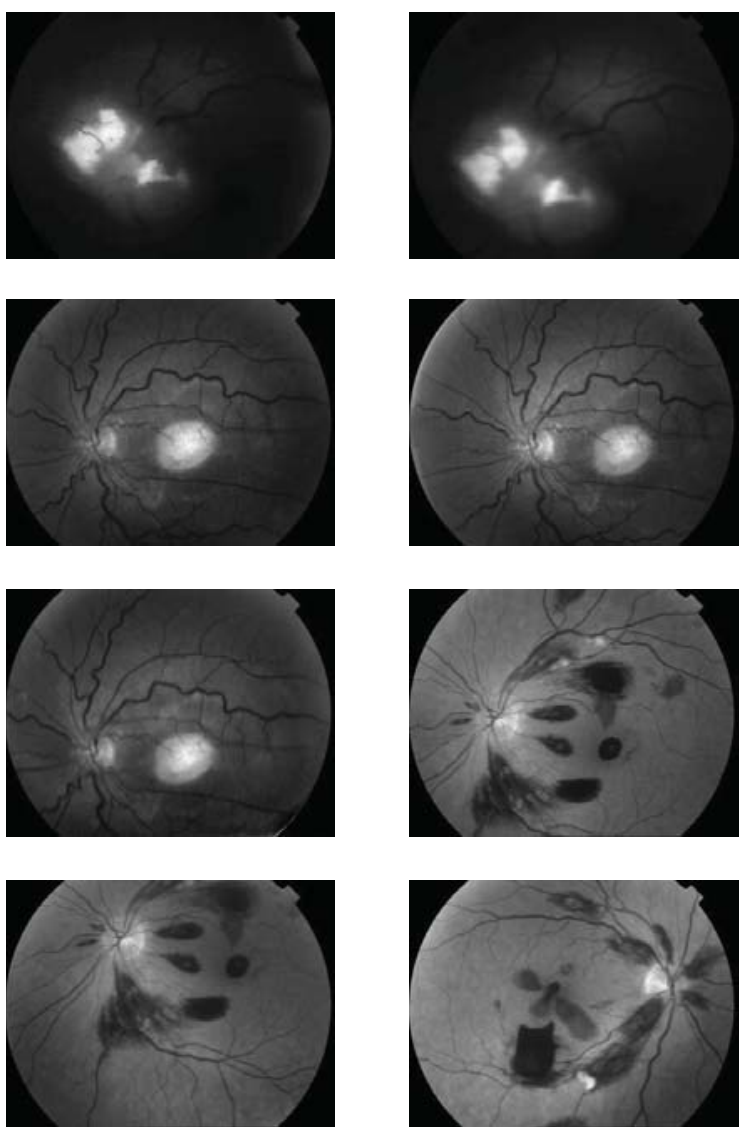

Fig. (3). Denoised Retinal Images by cycle spinning and ripplet transform. 

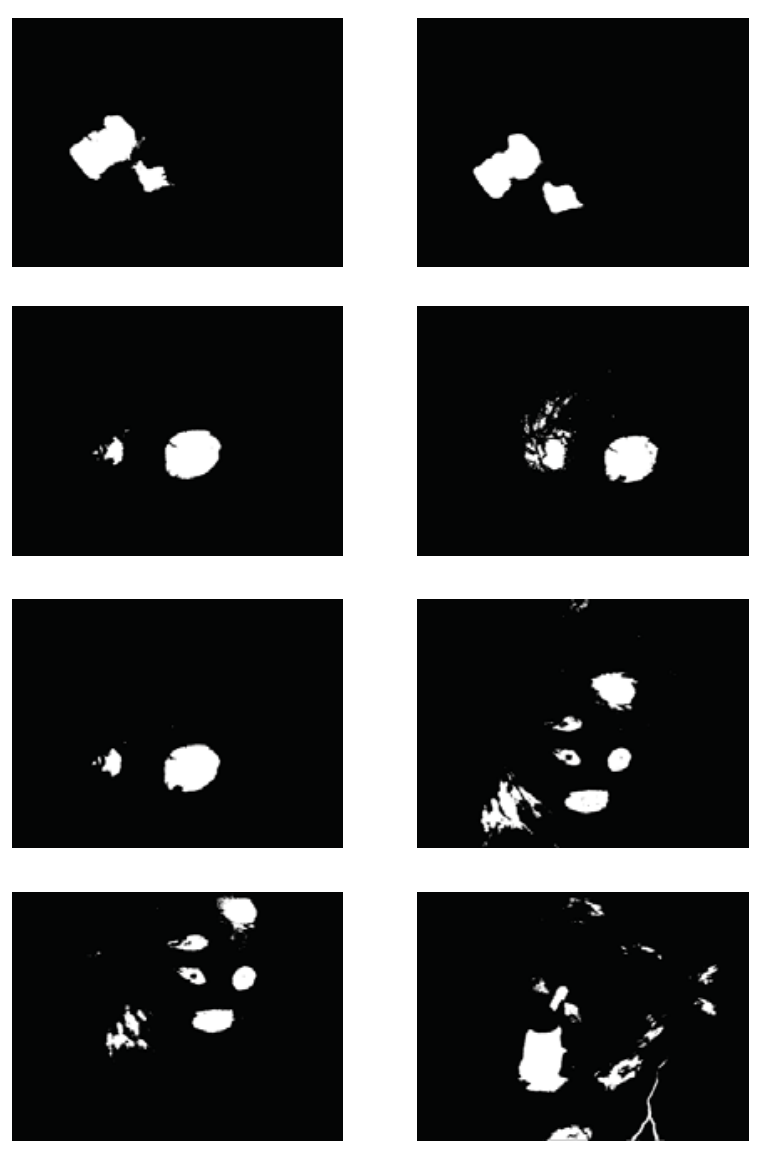

Fig. (4). Segmented Retinal Images by the FCLA after the denoising process while $\vartheta=0.02, \xi=0.02 \& \tau=50$.

Table 2. Statistical parameters for exudates detection process by the proposed method with and without denoising process.

\begin{tabular}{|c|c|c|c|c|c|c|c|c|c|}
\hline \multicolumn{10}{|c|}{$(\boldsymbol{\tau}=50)$ averaged over 50 images. } \\
\hline & & \multicolumn{4}{|c|}{ Without denoising } & \multicolumn{4}{|c|}{ With denoising } \\
\hline & $\delta \times \delta$ & $\begin{array}{l}\vartheta=0.005 \\
\xi=0.005\end{array}$ & $\begin{array}{l}\vartheta=0.01 \\
\xi=0.01\end{array}$ & $\begin{array}{l}\vartheta=0.02 \\
\xi=0.02\end{array}$ & $\begin{array}{l}\vartheta=0.05 \\
\xi=0.05\end{array}$ & $\begin{array}{l}\vartheta=0.005 \\
\xi=0.005\end{array}$ & $\begin{array}{l}\vartheta=0.01 \\
\xi=0.01\end{array}$ & $\begin{array}{l}\vartheta=0.02 \\
\xi=0.02\end{array}$ & $\begin{array}{l}\vartheta=0.05 \\
\xi=0.05\end{array}$ \\
\hline \multirow[t]{2}{*}{ Sensitivity } & $3 \times 3$ & $\sim 84 \%$ & $\sim 90 \%$ & $\sim 94 \%$ & $\sim 91 \%$ & $\sim 88 \%$ & $\sim 92 \%$ & $\sim 96 \%$ & $\sim 92 \%$ \\
\hline & $5 \times 5$ & $\sim 89 \%$ & $\sim 95 \%$ & $\sim 92 \%$ & $\sim 92 \%$ & $\sim 91 \%$ & $\sim 96 \%$ & $\sim 97 \%$ & $\sim 93 \%$ \\
\hline \multirow[t]{2}{*}{ Specificity } & $3 \times 3$ & $\sim 95 \%$ & $\sim 97 \%$ & $\sim 99 \%$ & $\sim 98 \%$ & $\sim 96 \%$ & $\sim 97 \%$ & $\sim 99 \%$ & $\sim 98 \%$ \\
\hline & $5 \times 5$ & $\sim 97 \%$ & $\sim 99 \%$ & $\sim 99 \%$ & $\sim 98 \%$ & $\sim 98 \%$ & $\sim 99 \%$ & $\sim 99 \%$ & $\sim 98 \%$ \\
\hline \multirow[t]{2}{*}{ Accuracy } & $3 \times 3$ & $\sim 88 \%$ & $\sim 92 \%$ & $\sim 94 \%$ & $\sim 94 \%$ & $\sim 89 \%$ & $\sim 94 \%$ & $\sim 96 \%$ & $\sim 95 \%$ \\
\hline & $5 \times 5$ & $\sim 92 \%$ & $\sim 94 \%$ & $\sim 96 \%$ & $\sim 92 \%$ & $\sim 92 \%$ & $\sim 95 \%$ & $\sim 97 \%$ & $\sim 92 \%$ \\
\hline \multirow[t]{2}{*}{ Average } & $3 \times 3$ & $\sim 89 \%$ & $\sim 93 \%$ & $\sim 95.7 \%$ & $\sim 94.3 \%$ & $\sim 91 \%$ & $\sim 94.3 \%$ & $\sim 97 \%$ & $\sim 95 \%$ \\
\hline & $5 \times 5$ & $\sim 92.7 \%$ & $\sim 96 \%$ & $\sim 95.7 \%$ & $\sim 94 \%$ & $\sim 93.7 \%$ & $\sim 96.7 \%$ & $\sim 97.7 \%$ & $\sim 94.3 \%$ \\
\hline
\end{tabular}

Table 3. Statistical parameters for exudates detection process by the proposed FCLA method for different rule updating parameters when $N^{\delta}=5 \times 5, \vartheta=0.01, \xi=0.01 \& \tau=30$.

\begin{tabular}{|c|c|c|c|c|c|c|}
\hline & & \multicolumn{5}{|c|}{ Updating Rules with Default $\rho=0.8$} \\
\hline & Fixed Rules & $\begin{array}{c}\bar{\eta}=0.5 \& \sigma \sigma= \\
0.02\end{array}$ & $\bar{\eta}=\underset{0.01}{0.6 \& \sigma}=$ & $\begin{aligned} \bar{\eta}= & 0.4 \& \sigma= \\
& 0.01\end{aligned}$ & $\bar{\eta}=\frac{0.6 \& \&}{0.05}=$ & $\bar{\eta}=\frac{0.4 \& \sigma}{0.05}=$ \\
\hline Sensitivity & $\sim 83 \%$ & $\sim 88 \%$ & $\sim 88 \%$ & $\sim 84 \%$ & $\sim 90 \%$ & $\sim 83 \%$ \\
\hline Specificity & $\sim 92 \%$ & $\sim 97 \%$ & $\sim 91 \%$ & $\sim 93 \%$ & $\sim 87 \%$ & $\sim 96 \%$ \\
\hline Accuracy & $\sim 84 \%$ & $\sim 89 \%$ & $\sim 83 \%$ & $\sim 87 \%$ & $\sim 81 \%$ & $\sim 81 \%$ \\
\hline Average & $\sim 86.3 \%$ & $\sim 91.3 \%$ & $\sim 87.3 \%$ & $\sim 88 \%$ & $\sim 86 \%$ & $\sim 86.7 \%$ \\
\hline
\end{tabular}


Table 4. Statistical parameters for exudates detection process by the proposed FCLA method for different threshold values of image binarization when $N^{\delta}=5 \times 5, \vartheta=0.01, \xi=0.01, \bar{\eta}=0.5, \sigma=0.02 \& \rho=0.8$.

\begin{tabular}{|c|c|c|c|c|c|c|c|c|c|}
\hline & \multicolumn{3}{|c|}{$\tau=\mathbf{3 0}$} & \multicolumn{3}{|c|}{$\tau=60$} & \multicolumn{3}{|c|}{$\tau=90$} \\
\hline & $\varsigma=0.3$ & $\varsigma=0.6$ & $\varsigma=0.9$ & $\varsigma=0.3$ & $\varsigma=0.6$ & $\varsigma=0.9$ & $\varsigma=0.3$ & $\varsigma=0.6$ & $\varsigma=0.9$ \\
\hline Sensitivity & $\sim 85 \%$ & $\sim 88 \%$ & $\sim 87 \%$ & $\sim 87 \%$ & $\sim 91 \%$ & $\sim 91 \%$ & $\sim 95 \%$ & 99\% & 97\% \\
\hline Specificity & $\sim 96 \%$ & $\sim 97 \%$ & $\sim 94 \%$ & $\sim 98 \%$ & $\sim 99 \%$ & $\sim 99 \%$ & $\sim 99 \%$ & $\sim 99.9 \%$ & $\sim 99.6 \%$ \\
\hline Accuracy & $\sim 87 \%$ & $\sim 91 \%$ & $\sim 89 \%$ & $\sim 89 \%$ & $\sim 95 \%$ & $\sim 94 \%$ & $\sim 97 \%$ & $\sim 99 \%$ & $\sim 98 \%$ \\
\hline Average & $\sim 89.3 \%$ & $\sim 92 \%$ & $\sim 90 \%$ & $\sim 91.3 \%$ & $\sim 95 \%$ & $\sim 94.7 \%$ & $\sim 97 \%$ & $\sim 99.3 \%$ & $\sim 98.2 \%$ \\
\hline
\end{tabular}

Table 5. Statistical parameters for exudates detection process by the proposed FCLA method compared to some other recent methods. For the FCLA method $N^{\delta}=5 \times 5, \vartheta=0.01, \xi=0.01, \tau=50, \bar{\eta}=0.5, \sigma=0.02, \rho=0.8 \& \varsigma=0.5$.

\begin{tabular}{|c|c|c|c|c|c|}
\hline & Method & NI/NIEx & Sensitivity & Specificity & Accuracy \\
\hline Zhang et al. [20] & SVM & $213 / 213$ & $88 \%$ & $84 \%$ & - \\
\hline Wang et al. $[17]$ & SVM & $154 / 54$ & $\mathbf{1 0 0 \%}$ & $71 \%$ & - \\
\hline Osareh [24] & FCMC & $300 / 300$ & $96 \%$ & $94.6 \%$ & - \\
\hline Garcia et al. [25] & MLP & $50 / 25$ & $84 \%$ & $62.7 \%$ & - \\
\hline Wisaeng et al. [26] & SVM & $120 / 98$ & $94 \%$ & $89.52 \%$ & - \\
\hline KNN & KNN & $50 / 20$ & $93 \%$ & $92 \%$ & $93 \%$ \\
\hline CLA [28] & CLA & $50 / 20$ & $96.3 \%$ & $98.7 \%$ & $96.1 \%$ \\
\hline Proposed method 1 & Hybrid & $50 / 20$ & $98.3 \%$ & $98.1 \%$ & $97.3 \%$ \\
\hline Proposed method 2 & Hybrid & $50 / 20$ & $\mathbf{9 9 . 4 \%}$ & $\mathbf{9 9 . 6 \%}$ & $\mathbf{9 9 . 1 \%}$ \\
\hline
\end{tabular}

\section{CONCLUSION}

In this paper, a hybrid method was presented for digital retinal image processing for diagnosis and screening purposes. A supervised/semi-supervised approach for exudate detection in fundus images was presented and the optimum structure was found in a comprehensive analysis. Ripplet transform and cycle spinning method was used to remove the noises and artifacts. The image was transformed into a fuzzy domain after it was denoised. A cellular learning automata model was used to detect any abnormality in the image, which is related to a lesion. STARE retina image dataset, for a neighborhood of $5 \mathrm{x}$ 5 , score of $\vartheta=0.01$, penalty of $\xi=0.01$, ratio of state overall variation in three sequent cycles in cellular automata $\bar{\eta}=0.5$, updating additive value $\sigma=0.02$ and rule selection threshold value $\rho=0.8$ the mean value of statistical criteria averaged over all dataset can reach $99 \%$ which is an outstanding assessment result for the proposed method. Considering the advantages and proficiencies of the proposed method, one may be interested in a more rapid algorithm for real-time applications where the processing should be accomplished in a short fraction of time. Hence, the authors suggest further studies and analyses of the computation time for the proposed method in the future.

\section{ETHICS APPROVAL AND CONSENT TO PARTI- CIPATE}

Not applicable.

\section{HUMAN AND ANIMAL RIGHTS}

No animals/humans were used for studies that are the basis of this research.

\section{CONSENT FOR PUBLICATION}

Not applicable.

\section{AVAILABILITY OF DATA AND MATERIALS}

Not applicable.

\section{FUNDING}

None.

\section{CONFLICT OF INTEREST}

The author declares no conflict of interest, financial or otherwise.

\section{ACKNOWLEDGEMENTS}

The authors would like to thank the staff and members of the research center, Birjand branch, Islamic Azad University, Birjand, Iran.

\section{REFERENCES}

[1] Walter T, Klein JC. Segmentation of color fundus images of the human retina: Detection of the optic disc and the vascular tree using morphological techniques InInternational Symposium on Medical Data Analysis.

[2] García M, Sánchez CI, Poza J, López MI, Hornero R. Detection of hard exudates in retinal images using a radial basis function classifier. Ann Biomed Eng 2009; 37(7): 1448-63.

[http://dx.doi.org/10.1007/s10439-009-9707-0] [PMID: 19430906]

[3] Singer DE, Nathan DM, Fogel HA, Schachat AP. Screening for diabetic retinopathy. Ann Intern Med 1992; 116(8): 660-71.

[http://dx.doi.org/10.7326/0003-4819-116-8-660] [PMID: 1546868]

[4] Sánchez CI, Hornero R, López MI, Aboy M, Poza J, Abásolo D. A novel automatic image processing algorithm for detection of hard exudates based on retinal image analysis. Med Eng Phys 2008; 30(3): 350-7.

[http://dx.doi.org/10.1016/j.medengphy.2007.04.010]

[PMID: 
17556004]

[5] Cree MJ, Olson JA, McHardy KC, Sharp PF, Forrester JV. The preprocessing of retinal images for the detection of fluorescein leakage. Phys Med Biol 1999; 44(1): 293-308. [http://dx.doi.org/10.1088/0031-9155/44/1/021] [PMID: 10071890]

[6] Pinz A, Bernögger S, Datlinger P, Kruger A. Mapping the human retina. IEEE Trans Med Imaging 1998; 17(4): 606-19.

[http://dx.doi.org/10.1109/42.730405] [PMID: 9845316]

[7] Patton N, Aslam TM, MacGillivray T, et al. Retinal image analysis: concepts, applications and potential. Prog Retin Eye Res. 25(1): 99-127.

[8] Eslami R, Radha H. The contourlet transform for image denoising using cycle spinning. InThe Thrity-Seventh Asilomar Conference on Signals, Systems \& Computers, 20032003 Nov 9 (Vol. 2, pp. 1982-1986). IEEE

[9] Akita K, Kuga H. A computer method of understanding ocular fundus images. Pattern Recognit 1982; 15(6): 431-43.

[http://dx.doi.org/10.1016/0031-3203(82)90022-X]

[10] Kochner B, Schulman D, Obermaier M, Zahlmann G, Mann G, Englmeier KH. A image processing system for analyzing color fundus photographs with regard to diabetic retinopathy. Klin Monatsbl Augenheilkd 1997; 211: 11.

[11] Li H, Chutatape O. Automated feature extraction in color retinal images by a model based approach. IEEE Trans Biomed Eng 2004; 51(2): $246-54$

[http://dx.doi.org/10.1109/TBME.2003.820400] [PMID: 14765697]

[12] Phillips R, Forrester J, Sharp P. Automated detection and quantification of retinal exudates. Graefes Arch Clin Exp Ophthalmol 1993; 231(2): 90-4.

[http://dx.doi.org/10.1007/BF00920219] [PMID: 8444365]

[13] Walter T, Klein JC, Massin P, Erginay A. A contribution of image processing to the diagnosis of diabetic retinopathy-detection of exudates in color fundus images of the human retina. IEEE Trans Med Imaging 2002; 21(10): 1236-43.

[http://dx.doi.org/10.1109/TMI.2002.806290] [PMID: 12585705]

[14] Ward NP, Tomlinson S, Taylor CJ. Image analysis of fundus photographs. The detection and measurement of exudates associated with diabetic retinopathy. Ophthalmology 1989; 96(1): 80-6.

[http://dx.doi.org/10.1016/S0161-6420(89)32925-3] [PMID: 2919052]

[15] Zahlmann G, Kochner B, Ugi I, et al. Hybrid fuzzy image processing for situation assessment. IEEE Eng Med Biol Mag 2000; 19(1): 76-83. [http://dx.doi.org/10.1109/51.816246] [PMID: 10659432]

[16] Goh KG, Hsu W, Lee ML. Automatic Diabetic Retinal Image Screening Using Image Processing and Machine Learning Algorithms. InSPIE Conference on Medical Imaging 2000.
[17] Wang H, Hsu W, Goh KG, Lee ML. An effective approach to detect lesions in color retinal images. InProceedings IEEE Conference on Computer Vision and Pattern Recognition. CVPR 2000 (Cat. No. PR00662) 2000 Jun 15. 2: 181-18. IEEE

[18] Gardner GG, Keating D, Williamson TH, Elliott AT. Automatic detection of diabetic retinopathy using an artificial neural network: a screening tool. Br J Ophthalmol 1996; 80(11): 940-4. [http://dx.doi.org/10.1136/bjo.80.11.940] [PMID: 8976718]

[19] Osareh A. Automated identification of diabetic retinal exudates and the optic disc (Doctoral dissertation, University of Bristol).

[20] Zhang X, Chutatape O. Top-down and bottom-up strategies in lesion detection of background diabetic retinopathy. 2005 IEEE Computer Society Conference on Computer Vision and Pattern Recognition (CVPR'05). 2: 422-8.

[http://dx.doi.org/10.1109/CVPR.2005.346]

[21] Walter T, Klein JC, Massin P, Erginay A. A contribution of image processing to the diagnosis of diabetic retinopathy--detection of exudates in color fundus images of the human retina. IEEE Trans Med Imaging 2002; 21(10): 1236-43.

[http://dx.doi.org/10.1109/TMI.2002.806290] [PMID: 12585705]

[22] Gardner GG, Keating D, Williamson TH, Elliott AT. Automatic detection of diabetic retinopathy using an artificial neural network: a screening tool. Br J Ophthalmol 1996; 80(11): 940-4.

[http://dx.doi.org/10.1136/bjo.80.11.940] [PMID: 8976718]

[23] Rosin PL. Training cellular automata for image processing. IEEE Trans Image Process 2006; 15(7): 2076-87.

[http://dx.doi.org/10.1109/TIP.2006.877040] [PMID: 16830925]

[24] Osareh A, Mirmehdi M, Thomas B, Markham R. Classification and localisation of diabetic-related eye disease. In European Conference on Computer Vision.

[http://dx.doi.org/10.1007/3-540-47979-1_34]

[25] Garcia M, Hornero R, Sánchez CI, López MI, Díez A. Feature extraction and selection for the automatic detection of hard exudates in retinal images. In2007 29th Annual International Conference of the IEEE Engineering in Medicine and Biology Society. Aug 22 (pp. 4969-4972). IEEE.

[http://dx.doi.org/10.1109/IEMBS.2007.4353456]

[26] Wisaeng K, Hiransakolwong N, Pothiruk E. Automatic detection of retinal exudates using a support vector machine. Appl Med Inform 2013; 32(1): 33-42.

[27] STARE Project Website Clemson University, Clemson, SC [online]. Available:. http://www.ces.clemson.edu/ ahoover/stare

[28] Nejad HC, Azadbakht B, Adenihvand K, Mohammadi M, Mirzamohammad M. Fuzzy cellular learning automata for lesion detection in retina images. J Intell Fuzzy Syst 2014; 27(5): 2297-303.

\section{C) 2019 Nejad et al.}

This is an open access article distributed under the terms of the Creative Commons Attribution 4.0 International Public License (CC-BY 4.0), a copy of which is available at: (https://creativecommons.org/licenses/by/4.0/legalcode). This license permits unrestricted use, distribution, and reproduction in any medium, provided the original author and source are credited. 